Preprint

\title{
Spectroscopic Measurements of Target Preheating on OMEGA
}

\author{
R.C. Elton, H.R. Griem and E.J. Iglesias ${ }^{a}$ \\ Institute for Plasma Research \\ University of Maryland, College Park, Maryland 20742
}

\begin{abstract}
The preheating of laser-heated microballoon targets has been measured by time-resolved $x$-ray and extreme ultraviolet (euv) spectroscopy on the $30 \mathrm{~kJ}, 351 \mathrm{~nm}, 60$-beam laser-fusion system at the University of Rochester Laboratory for Laser Energetics. Thin coatings of aluminum overcoated with magnesium served as indicators. Both the sequence of the $x$-ray line emission and the intensity of euv radiation were used to determine a preheating peaking at $\sim 10 \mathrm{~ns}$ prior to onset of the main laser pulse, with a power density $\$ 1 \%$ of the main pulse. The measurements are supported by numerical modeling. Further information is provided, by absorption spectra from the aluminum coating, backlighted by continuum from the heated surface. The exact source of the preheating energy remains unknown at present, but most likely arrives from early laser leakage through the system. The present target diagnostic is particularly useful when all beams cannot be monitored directly at all laser wavelengths.
\end{abstract}

PAC's: 52.50.Jm; $52.25 . \mathrm{Nr} ; 52.40 . \mathrm{Nk} ; 52.70 . \mathrm{La}$ 


\section{DISCLAIMER}

This report was prepared as an account of work sponsored by an agency of the United States Government. Neither the United States Government nor any agency thereof, nor any of their employees, make any warranty, express or implied, or assumes any legal liability or responsibility for the accuracy, completeness, or usefulness of any information, apparatus, product, or process disclosed, or represents that its use would not infringe privately owned rights. Reference herein to any specific commercial product, process, or service by trade name, trademark, manufacturer, or otherwise does not necessarily constitute or imply its endorsement, recommendation, or favoring by the United States Government or any agency thereof. The views and opinions of authors expressed herein do not necessarily state or reflect those of the United States Government or any agency thereof. 


\section{DISCLAIMER}

Portions of this document may be illegible in electronic image products. Images are produced from the best available original document. 


\section{INTRODUCTION}

Two related key issues in intertial confinement fusion (ICF) research are the uniformity and integrity of the irradiated spherical targets during surface heating and collapse. This is particularly important for the direct-drive approach, where the surface ablation and acceleration is achieved directly from the focused driving beams. Even small mass or density nonuniformities between layers undergoing mutual acceleration can be greatly amplified according to Rayleigh-Taylor ${ }^{1}$ hydrodynamic instabilities or, when shockaccelerated, by Richtmyer-Meshkov ${ }^{2}$ instabilities. Either can lead to pellet breakup prior to ignition. Greatcare is normally applied to forming targets of smooth surfaces. To avoid the imprinting of non-uniformities from the driving beam, considerable efforts are invested towards creating beams with irradiance uniformity within $1 \%$ variation or better on the target surface.

Design and numerical modeling usually are based upon the sudden heating of the target by the driving beam. Hence, any premature surface ablation that takes place prior to the onset of the main driving beam due to significant undesirable energy deposition could lead to a premature slow expansion of the ablator with an enlargement of surface area and decrease in density of the absorber, i.e., to a loss of target integrity. (This is separate from an early "foot" sometimes added prior to the beginning of the laser pulse, with a smoothness comparable to that of the main beam.) If not completely uniform, such a preheating could lead to the imprinting of auxiliary mass uniformities and hence instabilities. In the case of cryogenic targets (e.g., frozen deuterium), it could result in premature sublimation and interior cracking. Therefore, a large effort in laser design involves an enhancement of contrast, i.e., a ratio of laser-to- 
prepulse irradiance of perhaps five to six orders of magnitude. With laser drivers operating at a fundamental wavelength in the infrared and frequency upconverted into the ultraviolet, such contrasts are enhanced by a strong dependence of the conversion efficiency upon irradiance.

Such contrasts are routinely checked in laser beam design and development. However, when tens-to-hundreds of beams are involved in irradiating spherical ICF targets, it becomes impractical to routinely monitor the contrast for each beam for all wavelengths present. Instead, it becomes more prudent and neaningful to monitor emission arising from the target prior to the onset of the laser pulse as well as during the pulse to measure the contrast in deposited energy. A simple monitor of reflected light from the target surface at the laser wavelengths involved during the preheat period is a first approach; and indeed such preliminary results provide support for our interpretations. More definitive are time-resolved spectroscopic measurements which relate the emission to a specifically-identified target material (or materials in the case of layered coatings of varying substances). In this paper we describe such extremeultraviolet (euv) spectroscopic measurements ${ }^{3}$ of both emission and absorption from the preheating of multilayered coated spherical targets on the OMEGA laser-fusion system ${ }^{4}$ at the University of Rochester Laboratory for Laser Energetics (LLE). The techniques and interpretations described can be applied to other existing and future facilities.

\section{EXPERIMENT}

The experiments were carried out between May 1998 and April 1999 in three campaigns. The experimental layout is shown in Fig. 1. Fifty-nine beams from 
the "main" Omega laser system constituted the main-laser pulse at a wavelength of $351 \mathrm{~mm}$, with a combined energy of $20 \mathrm{~kJ}$ on target in a I ns Gaussian pulse. The beams were directed into the vacuum chamber of 3.3-m diameter and focused onto a spherical target of nominal overall diameter $940 \mu \mathrm{m}$, resulting in a peak irradiance of $-2 \times 10^{14} \mathrm{~W} / \mathrm{cm}^{2}$. Beam uniformity was provided by a combination of smoothing by spectral dispersion as well as by the use of distributed phase plates. Polymer microballoon targets of $20-\mu \mathrm{m}$ wall thickness were filled with neon to a pressure of $10 \mathrm{~atm}$, including a $10 \%$ admixture of argon as a tracer. A thin coating of aluminum $(0.03-\mu \mathrm{m}$ thick in May 1998 and earlier, reduced to $0.0125-\mu \mathrm{m}$ thereafter) was applied to the surface to help retain the gas. An additional laser shinethrough barrier (typically $0.1 \mu \mathrm{m}$ of aluminum) was not applied. Instead, a thicker ( 2 or $4 \mu \mathrm{m}$ ) outer coating of magnesium was added over the thin aluminum layer to form a tamper layer, also useful for spectroscopic diagnostics.

The euv diagnostics consisted of two 1-m grazing-incidence flat-field spectrographs ${ }^{5}$ covering the $30-300 \AA$ range with a spectral resolution of $0.1 \AA$. Both were equipped with a spherical collecting mirror near the slit. One of these was always equipped with a gated ( $\leqslant 1 \mathrm{~ns}$ ) four-stripline microchannel plate (MCP) for time-resolution, followed by photographic recording. Cables from these strips (of varying lengths to provide time delays) were properly matched and terminated at the MCP output to minimize pulse reflections. One of the spectrographs was mounted on the outside of the vacuum chamber with the collecting mirror at a distance of $210 \mathrm{~cm}$ from the target. The other spectrograph was mounted on a standard re-entrant platform ("TIM" in Fig. 1), with the collecting mirror $28 \mathrm{~cm}$ from the target for enhanced sensitivity. The use of such highly-selective (in wavelength) spectrographs was essential to 
discriminate against extraneous radiation when measuring large intensity ratios between the preheat and main phases of the event. In contrast, simpler thinmetal bandpass-filtered euv detectors alone can transmit x-rays freely above a certain photon energy at the high temperatures encountered in the main laserplasma event; while only measuring euv emission in a cooler preheater phase. This can result in a large underestimate of the relative preheat euv emission.

We also had available at LLE a soft $x$-ray, 100-cm radius, convex-curved crystal (KAP) spectrograph set to measure some of the magnesium and aluminum hydrogenic (Mg XII, AI XIII) and helium-like (Mg XI, AI XII) resonance lines in the 6.4-8.6 $\AA(1.4-1.9 \mathrm{keV})$ range. This instrument was equipped with a streak camera and photographic readout for temporal resolution. Since this spectrograph is slitless, the spectral resolution is sourcesize limited; this feature provided a measure of the spatial extent of the plasma, as determined from the spectral line widths. Also fielded were various LLEprovided $x$-ray imaging cameras with varying degrees of filtration, and operating in both the time-resolved and time-integrated modes.

\section{OBSERVATIONS}

The first evidence of significant preheating of the target arose following the first two shots taken in May 1998, i.e., in a rather sudden change of events that continued throughout that campaign. On those first two shots (both at $\sim 14 \%$ lower energy than for the shots that followed), the temporal evolution shown in the $x$-ray streak spectral data was typical of those obtained in previous campaigns, in that intense and narrow magnesium resonance spectral lines arising from the heating of the outer layer preceded in time those from the thin 
aluminum inner layer. Such a time-resolved spectrum is shown in Fig. 2 and is considered "normal", such as we had observed during several previous campaigns at LLE. The magnesium spectral lines are more intense than those from aluminum because of the much thicker coating of the former. Resonance series lines up to $n=5$ (transitioning to $n=1$ ) are distinct for both helium-like and hydrogenic magnesium. The narrowest aluminum and (initial) magnesium lines corresponds to $\sim 1 \mathrm{~mm}$ at the target, i.e., approximately the original target size.

In stark contrast to the first two shots, data obtained similarly on the succeeding 19 shots in this campaign showed broadened aluminum lines already at the onset of the laser pulse, accompanied or followed by quite diffuse (broadened) magnesium lines of less intensity. An example of such an "alternate" spectrum is shown in Fig. 3. In such spectra, the magnesium lines are expanded to the extent that series members above $n=2$ are barely distinguishable. The predominance of the aluminum lines indicates that most of the magnesium has been vaporized and that the laser directly heats the aluminum undercoating. Hence, the aluminum lines also become broadened due to expansion.

The $\mathrm{Mg} X I I n=2$ to $n=1$ Lyman- $\alpha$ line appears to be composed of two lines, which is associated with an already expanded shell of $\sim 4 \mathrm{~mm}$ diameter at the onset of the main laser pulse, i.e., having experienced an $\sim 1.5-\mathrm{mm}$ radial expansion of the surface during a preheat period of $10 \mathrm{~ns}$ (from the euv data discussed below). This results in a radial expansion velocity of $\sim 1.5 \times 10^{7} \mathrm{~cm} / \mathrm{sec}$, which is not unreasonable. This measured velocity agrees with numerical modeling of the initial blowoff towards the laser, using the FAST radiation transport hydrocode $e^{6}$ for spherical geometry, and assuming an overall 
preheating irradiance on target of $3 \times 10^{12} \mathrm{~W} / \mathrm{cm}^{2}$ (as inferred below from the euv data). ${ }^{7}$ There is also experimental evidence of a narrow central ine, presumably from the target surface, which indicates that some of the coatings continue to be relatively intact. This is also consistent both with late-time data following compression and with modeling of the target surface area, as described below.

Also present (and not observed previously at all on more "normal" shots) at a time of $\sim 0.7$ ns after onset of the main pulse and persisting for $\sim 0.2 \mathrm{~ns}$ was the $\mathrm{K}_{\dot{\alpha}}$ line formed following innershell ionization in neutral (and slight! ?-ionized) aluminum at a wavelength of $8.34 \AA$. This suggests the presence of fast electrons often associated with instabilities, and perhaps target breakup in the form of both hot spots and cold aluminum, since the $K_{\alpha}$ line is observed concurrently with resonance lines of AI XII and AI XIII. (A much weaker $K_{\beta}$ line at $7.96 \AA$ was also detected on one shot, adding confirmation to the identification of the $\mathrm{K}_{\alpha}$ line.)

Coincident with the $x$-ray observations of an apparent preheating and vaporization of the target coatings was the corresponding observation of early euv spectra that included continuum as well as line emissions arising from $n=3$ to $n=2$ transitions, mostly of lithium-like $\mathrm{Mg} X$ spectra, but also of less intense helium-like and hydrogenic Mg XI and XII spectra, respectively. The latter emissions from the higher ionization stages peaked at $\sim 10$ ns before the onset of the main laser pulse, giving way to $\mathrm{Mg} X$ (and lower) spectra in the last $5 \mathrm{~ns}$. (Similar emissions from the 70-times thinner aluminum layer are expected to be much less intense, with some unfortunate wavelength overlaps, and are difficult to distinguish.) Also observed prior to the main laser pulse onset were carbon lines, presumably arising from: (a) the microballoon surface following localized penetration, (b) the target surface as contaminants, or (c) the 10- $\mu \mathrm{m}$ diameter 
supporting stalk. They showed a dominance of $C V I$ over $C V n=2$ to $n=1$ resonance lines.

Such preheating euv spectral features are illustrated in the temporal plot of overall emission in the 100-200 $\AA$ wavelength region, shown in Fig. 4 from multiple shots during the May 1998 campaign, using four MCP gated striplines for temporal resolution. This indicates a preheating euv emission of $\sim 0.7 \%$ of that of the corresponding continuum spectrum observed during the main event. This suggests a power flux deposited at the target of $\sim 3 \times 10^{12} \mathrm{~W} / \mathrm{cm}^{2}$ in the preheat period, which was used as input for the numerical modeling ${ }^{6}$ discussed elsewhere here.

That an irradiance of this magnitude could produce some $\mathrm{Mg}$ XI and XII euv emission is evidenced experimentally by a distinct $x$-ray streak spectium obtained at the lowest irradiance used at LLE, namely $7 \times 10^{12} \mathrm{~W} / \mathrm{cm}^{2}$. Additionally, recent experiments at Darmstadt ${ }^{\S}$ with a laser-irradiated magnesium slab target produced an x-ray spectrum that included Mg XI and XII at an irradiance as low as $1 \times 10^{12} \mathrm{~W} / \mathrm{cm}^{2}$. (Again, one cannot rule out hot spots as a source of radiation from these species, particularly when $K_{\alpha}$ lines are observed, as described above.) As with the x-ray streak spectra, the euv preheating began on the third shot in the May 1998 campaign, i.e., no euv spectral emission was measured on the two striplines set for early timing on the first two shots (the remaining two striplines timed later did record the main event, which verified proper operation of the detector). This again suggests some sudden event after the first two shots, resulting in target preheating. 
The C VI and $\mathrm{Mg} X$ spectral lines observed at the peak of the preheating period shown in Fig. 4 signify an electron temperature of $k T_{e} \sim 100-150 \mathrm{eV}$, which again is consistent with numerical computations ${ }^{6}$ for the same pulse shape ${ }^{9}$ as shown in Fig. 4 and using an overall target irradiance of $3 \times 10^{12} \mathrm{~W} / \mathrm{cm}^{2}$, as inferred above. Also, the calculations predict an expanding (towards the laser) plasma consisting of ions dominated by charge states of $\mathrm{Mg}^{9+}$ (Li-like) and $\mathrm{Mg}^{10+}(\mathrm{He}-\mathrm{like})$ at the peak of the preheating pulse. This accounts for the Li-like nd-2p emission from recombination (as well as excitation). Excitation of line emission in the He-like and $\mathrm{H}$-like ions during this period most likely occurs in hot spois (as supported again by $\mathrm{K}_{\alpha}$ emission). A measured decline to a predominantly $\mathrm{Li}$-like $\mathrm{Mg} X$ spectral feature in the 4-5 ns prior to main laser pulse onset is consistent with numerical predictions of a temperature drop to a few $10^{\prime}$ s of $\mathrm{eV}$ and charge states ranging from $\mathrm{Mg}^{6+}$ to $\mathrm{Li}$-like $\mathrm{Mg}^{9+}$ during that period. This is followed by a reheating of residual magnesium at main laser pulse onset, once again producing $\mathrm{Mg} X \mathrm{X}$ and $\mathrm{XII}$ line emissions in a collapsing plasma.

The numerical modeling of density, using the pulse in Fig. 4, also predicts at least a partial breakdown in target integrity beginning at $\sim 3-4 \mathrm{~ns}$ after onset of the preheat, followed by a heat wave flowing through the $20-\mu \mathrm{m}$ thick microballoon wall into the gas filling to a depth of $\sim 100 \mu \mathrm{m}$ at the time of onset of the main laser pulse. This breakup could be contributing to the decrease in euv emission shown in Fig. 4 beginning at $\sim 8 \mathrm{~ns}$ prior to main laser onset. However, a boundary between the $\mathrm{CH}$ and the magnesium also is predicted to be somewhat sustained, along with some residual magnesium available for reheating at main laser pulse onset, as observed. All modeling ${ }^{6,7}$ predicts a critical-density layer traveling inward during the preheating phase. Since this 
layer is at a density much less than that for a solid, it indicates once again that surface material such as magnesium has expanded away.

That the weaker euv spectrum observed in the preheat period is not simply later-time emission recorded by a weak secondary pulse to the MCP striplines has been verified experimentally by the vast difference in the simple, few-line, few-species spectra obtained at early times compared to a multi-line spectrum from recombination to many species at the later times, including Ne VIII (Li-like) $2 p-3 d$ emission at $98 \AA$ from the core filling, not present in the preheated plasma. Another distinct aspect of the late-time recombination spectra is a ratio of C VI to $\mathrm{CV}$ resonance lines of order unity, signifying a temperature of $\mathrm{kT}_{\mathrm{e}} \sim 75 \mathrm{eV}$, lower than at the peak of the preheating phase as reported above. Hence, this alternative explanation for the preheater spectra observed is first ruled out on the basis of non-time-correlated spectral features. Furthermore, a measured ratio of peak voltages between the secondary and primary pulses of $\sim 1 / 5$ results in a MCP gain ratio of $10^{-8}$, using a power-law gain dependence ${ }^{10,11}$ on applied stripline voltage $V$ as strong as $V^{11}$, thereby resulting in a negligible gain on the secondary pulse.

A cause for this sudden onset of preheating could not be established at the time of the May 1998 campaign. During the October 1998 campaign, which included 21 shots, the anomalies in the x-ray streak spectral data were not readily apparent. The euv preheat emission, while still present with the same ionic species, was $\sim 1 / 4$ of that measured in May. These data indicated that the preheating effects had diminished somewhat, perhaps associated with improved binding of the magnesium with the use of aluminum layers of less than one-half the thickness. 
A further understanding of the preheating mechanism at the target arose from the third campaign, carried out in April 1999. For this limited series of 10 shots, the closer-in and hence more sensitive TIM-mounted euv spectrograph was equipped with the time-gated MCP detector: Again, the aluminum inner layer was thin $(0.0125-\mu \mathrm{m})$. For this campaign, the $2-\mu \mathrm{m}$ thick magnesium outer layer was overcoated with $2 \mu \mathrm{m}$ of $\mathrm{CH}$ to provide additional tamping. Also, some $2-\mu \mathrm{m}$ thick $\mathrm{LiF}$-coated targets (without $\mathrm{CH}$ overcoatings) were used. The results to be described next were similar for all of these target designs.

The euv data in this campaign showed overall continuum emission during the preheat phase equivalent to that from the October 1998 campaign. Particularly obvious in this series of experiments were a number of absorption features in the euv spectra during the preheating phase which were not present during the main event. These are shown in the spectrum in Fig. 5. This attests to the "purity" of the euv continuum, i.e., not being an accumulation of higher orders and scattered light of other wavelengths which would not have been absorbed in these lines. The strongest and broadest absorption feature (at a wavelength of $116 \AA$ ) matches a blend of the energies ${ }^{12}$ for photoionization from the $2 s$ innershell levels in neutral aluminum as well as in $\mathrm{Al}^{1+}$ and $\mathrm{Al}^{2+}$ ions. In addition, photoionization edges ${ }^{13}$ associated with valence electrons in $\mathrm{Al}^{3+}$ to at least $\mathrm{Al}^{5+}$ match other narrower absorption features in the spectra, included in Fig. 5. (That 2 s-innershell absorption at $156 \AA$ did not appear for magnesium is another indication that the magnesium coating vaporizes and expands at early times in the preheat phase.) 
One interpretation of such absorption features is that they arise from a continuum background formed at the front surface of the CH microballoon (the balloon wall as well as the gaseous fill are opaque in this region, ruling out the possibility of a rear-target-surface backlighting, unless one invokes major target breakup). The $0.0125-\mu \mathrm{m}$ thick solid aluminum sealant layer is semi-transparent $(\sim 25 \%)$ to the $351-\mathrm{nm}$ wavelength laser light, $1 \frac{1}{4}$ while also being semi-opaque ( $\sim 40 \%$ absorption) at the at the $x$-ray edges. ${ }^{15}$ As such, laser preheater light can be transmitted through the thin aluminum layer to the outer surface of the polymer target, generating a continuum backlighter, and forming the $2 \mathrm{~s}$ absorption feature in the aluminum layer. This holds similarly for $n=2$ absorption in an aluminum plasma that might be formed such that the product of density and thickness remains constant over a limited range. In addition, the absorption features from multiple ionization stages could be arising from different points on the target, and perhaps from varying thicknesses of the aluminum layer, if it is not completely uniform in the case of hot spot formation.

Alternatively, or additionally, it is possible that the absorption is forming along chords in the "limb" of the spherical shell of aluminum, rather than through the layer itself. This would increase the absorption length by perhaps a factor-of-10 compared to that for absorption perpendicular to the layer. In this scenario, the continuum backlighting could be arising from a heated coating, as well as the $\mathrm{CH}$ surface.

In campaigns that took place earlier than that of April 1999, only a close examination of the October 1998 data taken with the more remote spectrograph showed a weak presence of the 2 s-innershell absorption band. This marked difference may have been a result of a different mounting position on the 
spherical target chamber for the April 1999 campaign, being almost opposite to the earlier ones. This could be interpreted as an effect of certain selective beams being involved in the preheating, rather than all 59 uniformly. This could also have contributed to the backlighting and absorption along a chord.

Turning to the x-ray streak spectra obtained during this April 1999 campaign, a very sensitive fresh CSI photocathode was in place on the $x$-ray streak spectrograph for two shots. As such, the late-time spectra were very overexposed. However, a close inspection of the films obtained indicate some evidence of a low-intensity Mg XII Ly- $\alpha$ line for approximately $1 \mathrm{~ns}$ prior to the main pulse. The maximum preheater emission at $-10 \mathrm{~ns}$, expected from the euv data, could not be detected on these two shots because of a limited sweep range, and remains a topic for future experiments.

\section{CONCLUSIONS}

The early-time spectrographic data presented here are consistent with a numerical model of low-energy preheating in regions of sufficient energy deposition in the target to produce Mg X (and XI and XII in spots) spectra and premature evaporation and subsequent expansion of the outer coating, along with a loss of target integrity. Transmission of this beam through the thin aluminum sealant layer leads to a preheating of the target surface, thereby producing CV and VI emission along with a continuum that backlights the aluminum and produces observed absorption features, either perpendicular to the layer surface or through chords in the shell, along the axis of view, or perhaps both. 
An obvious explanation for the target preheating is that there is a relatively weak laser prepulse occurring at early times, if not from the ultraviolet $(0.351$ $\mu \mathrm{m}$ wavelength) beam, perhaps of a fundamental- $(1.053 \mu \mathrm{m})$ or doubled- $(0.527$ $\mu \mathrm{m})$ equivalent frequency not completely converted. Other experiments as well as numerical modeling now show that a projected irradiance of $\sim 10^{12} \mathrm{~W} / \mathrm{cm}^{2}$ is sufficient to produce the observed features. If indeed the preheating arises from laser beams, such heated zones would represent severe non-uniformities in the beam profiles and could result in laser imprinting at the beginning of the main pulse, thereby generating instabilities as mentioned earlier here and breakdown of target integrity throughout. Also, excessive preheating of the low mass $\mathrm{CH}$ surface adjacent to the aluminum layer could produce pressure differences to further drive instabilities. It will be very interesting to obtain additional measurements of the preheating pulse shape for a more exact modeling of the effects.

The source of the energy for the observed target preheating remains under investigation, as does the wavelength (i.e., fundamental, frequency doubled or tripled), should it be associated with the OMEGA laser. The power of this target technique in diagnosing a prepulse and the overall contrast with the main beam when multiple beams are present is demonstrated. The fact that this preheating began as a sudden event which may have subsided somewhat over time indicates the importance of a thorough understanding and continuing monitoring of the energy reaching the target, lest it reoccur randomly during future experiments. 


\section{ACKNOWLEDGEMENTS}

a) Permanent address: Departamento de Fisica, Universidad Simón Bolivar,
Caracas, Venezuela

Extensive cooperation of the scientific, laser engineering and technical staffs at the University of Rochester Laboratory of Laser Energetics in obtaining the data described is recalled with appreciation. Numerical modeling by J.P. Dahlburs and colleagues at NRL added significant support to the interpretation of the data. This work was supported by the U.S. Department of Energy as part of the National Laser User Facility (NLUF) program. 


\section{REFERENCES}

1. S. Chandrasekhar, in Hydrodynamics and Hydromagnetic Stability, Chapter 10 (Oxford University Press, 1968), analyzed from the originals in: Lord Rayleigh, Scientific Papers, ii, 200 (Cambridge, England, 1900) and G. Taylor, Proc. Roy. Soc. (London) A, 201, 192 (1950).

2. R.D. Richtmyer, "Shock Wave Instabilities", Communication of Pure and Applied Mathematics 13, 297 (1960) (theory); and E.E. Meshkov, Fluid Dynamics 4 (5) 101 (1969) (experiment). See also R.L. Holmes, et al., J. Fluid Mech. 389, 55 (1999).

3. R. Elton, E. Iglesias, H. Griem, G. Pien, D. Bradley, J. Delettrez and R. Epstein, "Early-Time Extreme-UV Emission from OMEGA Plasmas", Bull. Am. Phys. Soc. 43, 1646 (1998); also R. Elton, H. Griem and E. Iglesias, "Further Evidence for a Prepulse on OMEGA", Bull. Am. Phys. Soc. 44, 38 (1999).

4. R.S. Craxton, et al., "OMEGA Upgrade Design", Laboratory for Laser Energetics Report DOE/DP 40200-101, University of Rochester (1989); also W. Seka, S.D. Jacobs, J.E. Rizzo, R. Boni and R.S. Craxton, Opt. Commun. 34, 469 (1980); and R.S. Craxton, IEEE J. Quantum Electron. QE-17, 1771 (1981).

5. T. Kita, T. Harada, N. Nakano and H. Kuroda, Applied Optics 22, 512 (1983) and 23, $2386(1984)$. 
6. J.P. Dahlbury, J.H. Gardner, M. Klapisch and D. Colombant, Naval Research Laboratory, private communication, 1999. The FAST radiative transport compressible hydrocode for spherical geometry utilizes tabulated equations. of state, Spitzer thermal conduction, inverse bremsstrahlung laser cleposition and accounts for non-LTE conditions using the Busquet model. Opacities are computed with the Super Transition Array code. For further details see J.H. Gardner, A.J. Schmitt, J.P. Dahlburg, C.J. Pawley, S.E. Bodner, S.P. Obenschain, V. Serlin and Y. Aglitskiy, Phys. of Plasmas 5, 1935 (1998).

7. Early encouraging numerical estimates by J.A. Delettrez at LLE using $1 / 3$ this irradiance are consistent with the detailed NRL modeling described here.

8. F. B. Rosmej, Technische Universität Darmstadt, private communication, 1999; to be published.

9. The measured euv emission shown in Fig. 4 is somewhat sensitive to plasma evolution and may not entirely reflect the true pulse shape of the preheating impulse used in the modeling, particularly after the first 5 ns of target integrity. However, preliminary evidence from a target-reflected laser light measurement indicates that it is approximately correct during that period, even though longer than the Omega pulse.

10. E.H. Eberhardt, Applied Optics 18, 1418 (1979).

11. C.J. Pawley and A.V. Deniz, "Improved Measurements of Noise and Resolution of X-ray Framing Cameras at 1-2 keV", Rev. Sci. Instruments (in press). 
12. W.D. Barfield, G.D. Koontz and W.F. Huebner, J. Quant. Spectros. Radiat. Transfer 12, 1409 (1972).

13. R.L. Kelly, J. Phys. Chem. Ref. Data 16, Suppl. 1, 1987.

14. American Institute of Physics Handbook, Third Edition, D.E. Gray coordinating editor, (McGraw-Hill Book Co., New York, 1972), p. 6-159, using optical constant data from G. Hass and J.E. Waylonis, J. Op Soc. Am. $50,1133(1960)$.

15. B.L. Henke, E.M. Gullickson and J.C. Davis, Atomic Data and Nuclear Data Tables 54, 181 (1993). 


\section{FIGURE CAPTIONS}

Fig. 1. Layout of the experiments. "TIM" refers to the ten-inch manipulator reentrant platform upon which one of the euv spectrographs was mounted.

Fig. 2. X-ray streak spectrum typical of those obtained for the first two shots in the May 1998 and in previous campaigns, showing the expected sequence of $\mathrm{Mg}$ XI (He-like) and Mg XII (H-like, Lyman series) resonance lines followed by similar AI XI, and AI XIII lines, from oute, and inner layers, respectively.

Fig. 3. X-ray streak spectrum typical of those obtained after the first two shots in the May 1998 campaign, showing the early onset of aluminum lines from the inner layer. This shows the dominance of Al XII (He-like) and Al XIII (H-like Lyman series) lines from the inner layer at early times.

Fig. 4. Logarithmic time history of euv emission in the 100-200 $\AA$ wavelength range. The data points shown and connected by a smooth curve were obtained from various shots and striplines used in the May 1998 campaign, with normalization between strips obtained from pointing shots taken with gold targets. Corrections have been applied for film saturation. The $\sim 4$ ns duration of emission during the main pulse agrees with that of continuum emission in the $x-$ ray streak spectra (not readily apparent in the copy in Fig. 3), possibly even including here (but not curve-fitted) a decrease over an interval of $\sim 0.5$ ns at 3 ns from the onset of the main laser pulse. 
Fig. 5. Euv spectra obtained from two adjacent MCP striplines during the April 1999 campaign, indicating some main emission as well as absorption features.

The carbon emission is most likely associated with target surface vaporization. 


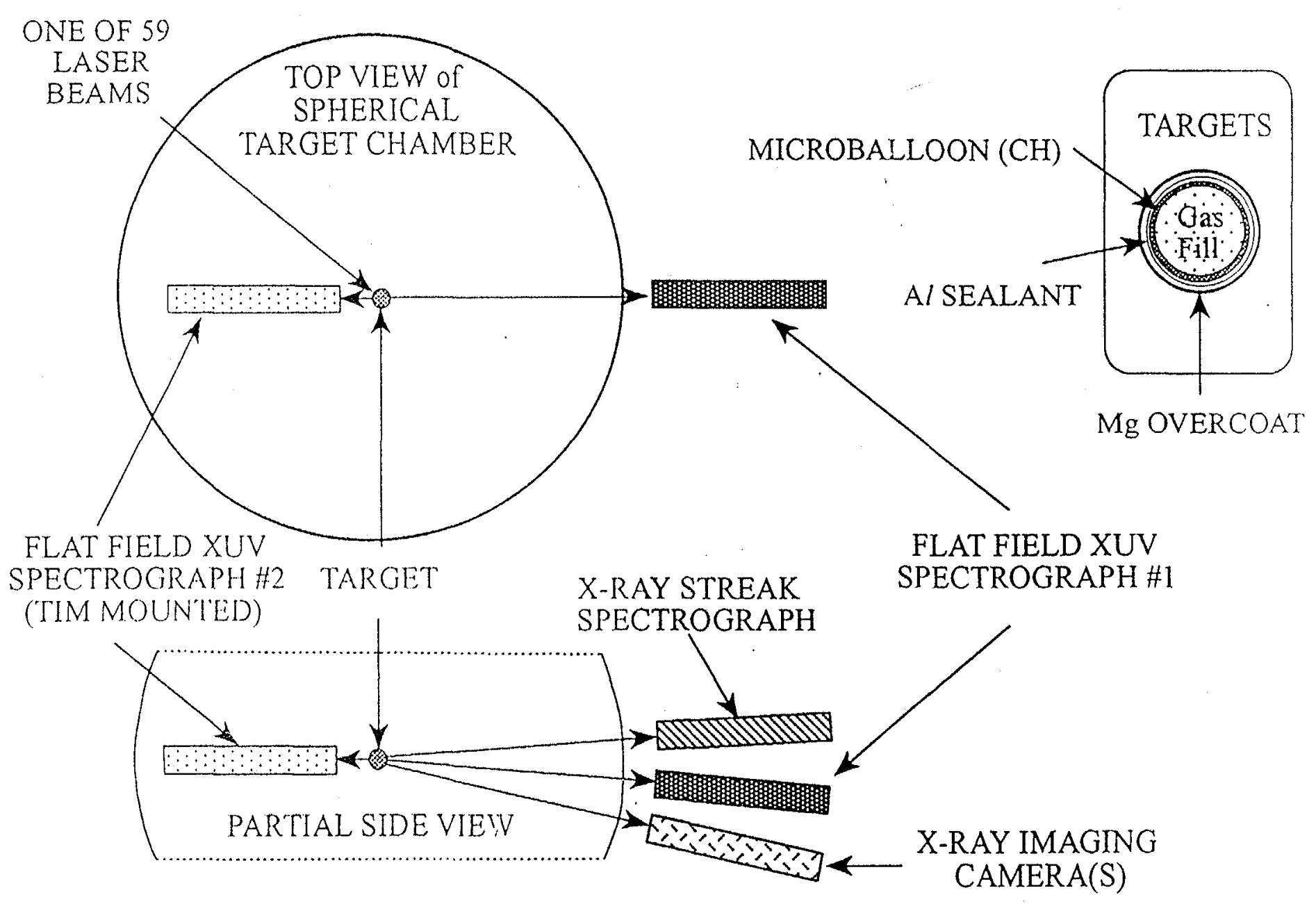

Fig. 1. Layout of the experiments. "TIM" refers to the ten-inch manipulator reentrant platform upon which one of the euv spectrographs was mounted. 


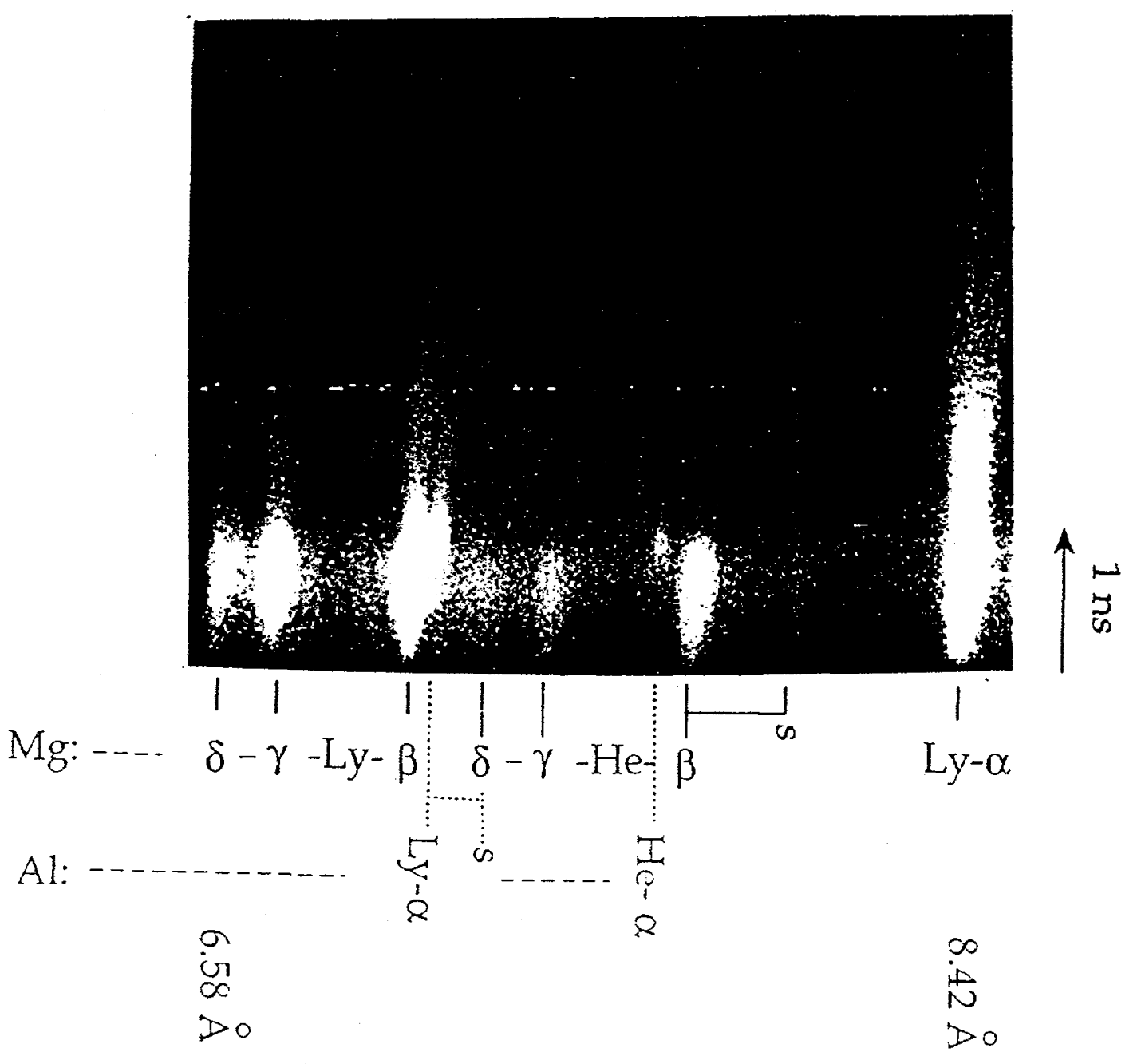

Fig. 2. X-ray streak spectrum typical of those obtained for the first two shots in the May 1998 and in previous campaigns, showing the expected sequence of $\mathrm{Mg}$ $X I$ (He-like) and Mg XII (H-like, Lyman series) resonance lines followed by similar AI XII and AI XIII lines, from outer and inner layers, respectively. 

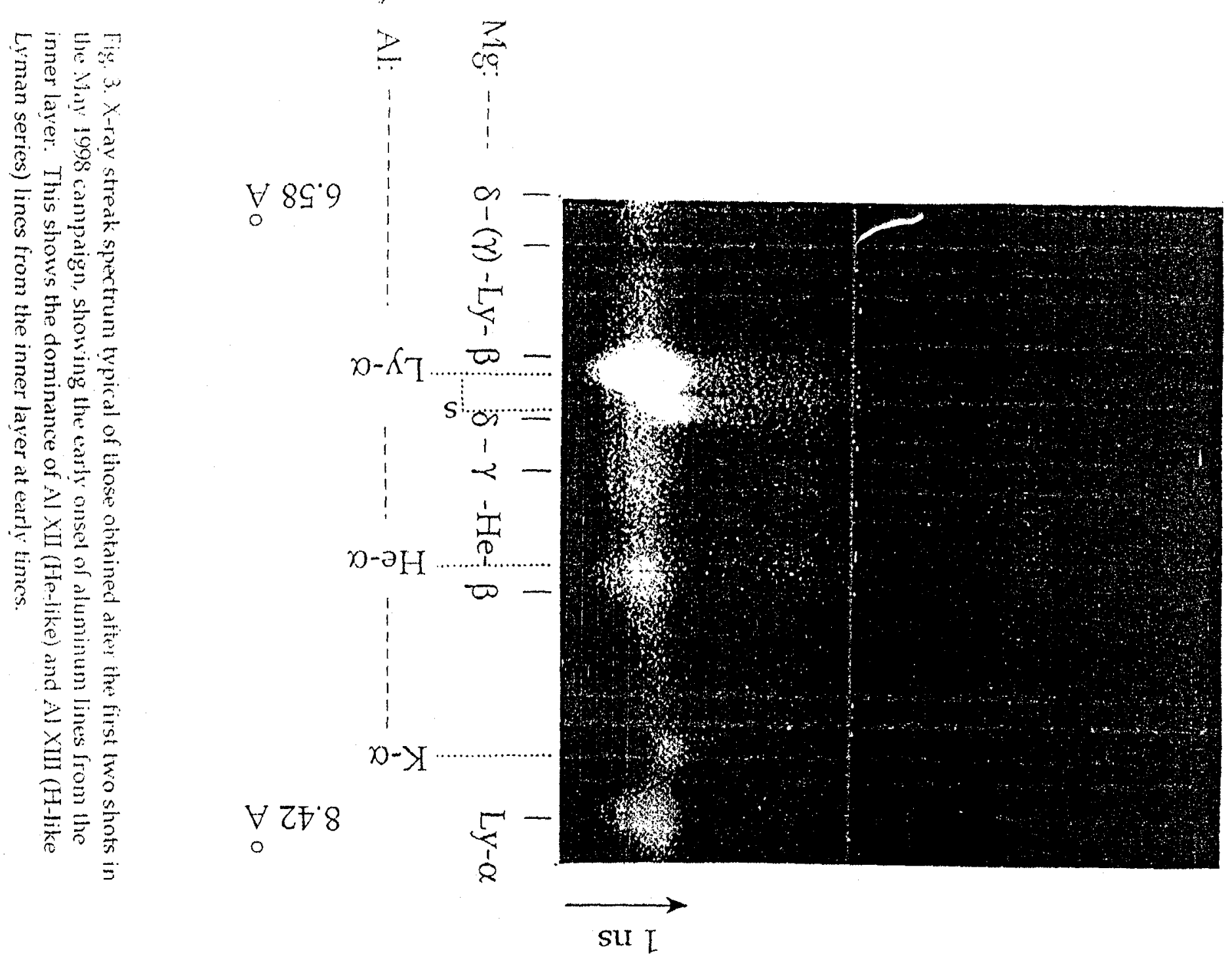


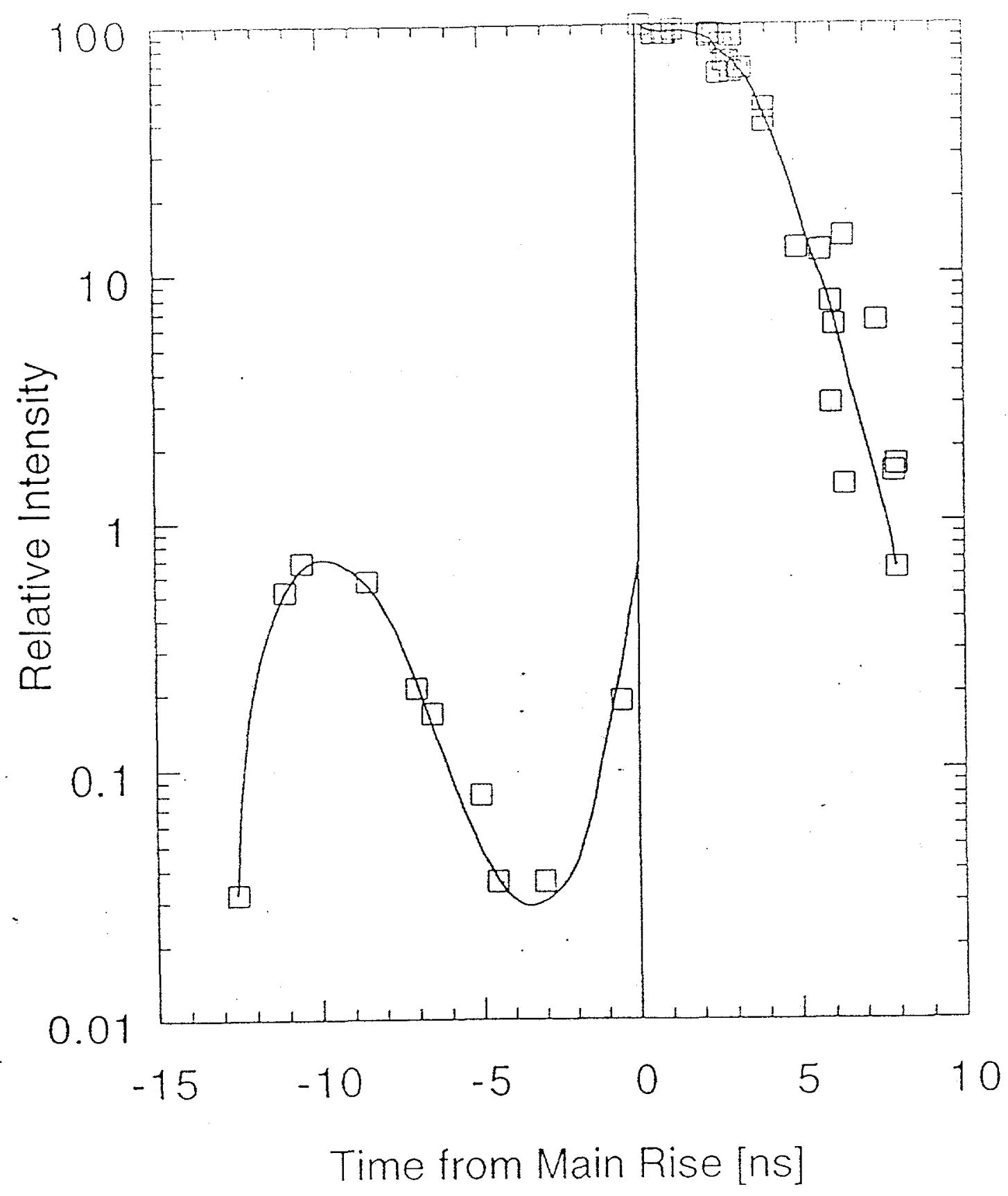

Fig. 4. Logarithmic time history of euv emission in the 100-200 $\AA$ wavelength range. The data points shown and connected by a smooth curve were obtained from various shots and striplines used in the May 1998 campaign, with normalization between strips obtained from pointing shots taken with gold targets. Corrections have been applied for film saturation. The $\sim 4 \mathrm{~ns}$ duration of emission during the main pulse agrees with that of continuum emission in the $x$ ray streak spectra (not readily apparent in the copy in Fig. 3), possibly even including here (but not curve-fitted) a decrease over an interval of $\sim 0.5 \mathrm{~ns}$ at $3 \mathrm{~ns}$ from the onset of the main laser pulse. 


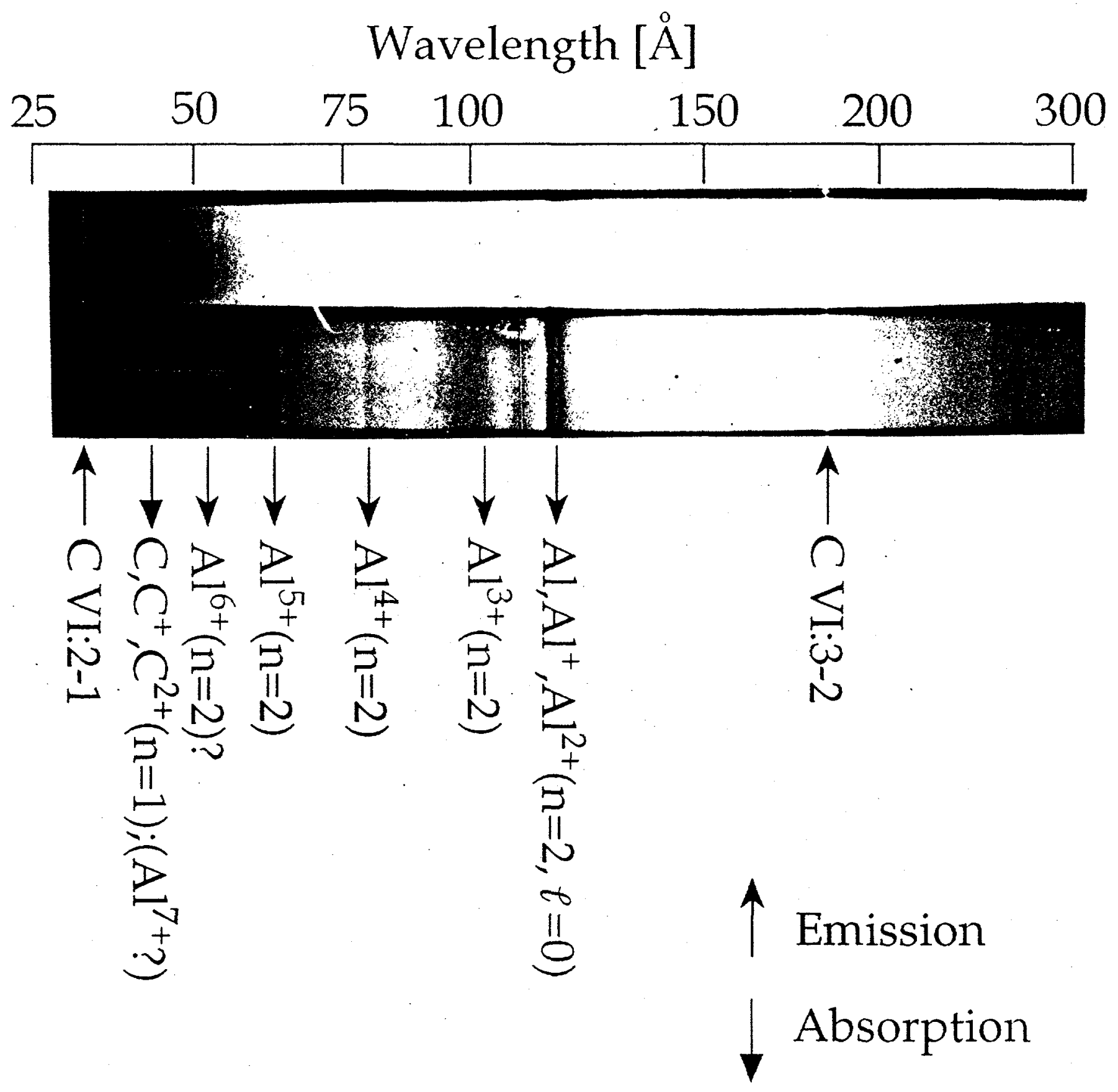

Fig. 5. Euv spectra obtained from two adjacent MCP striplines during the April 1999 campaign, indicating some main emission as well as absorption features. The carbon emission is most likely associated with target surface vaporization. 